\title{
Prevalence of Tobacco Use and Associated Factors among Adults in Benin in 2015: Results of the National Survey of Non-Communicable Disease Risk Factors
}

\author{
Alexandrine Houinato ${ }^{1}$, Roch Christian Johnson', Alphonse Kpozehouen ${ }^{2}$, Fernand Guedou ${ }^{3}$, \\ Christophe Houssou ${ }^{4}$, Dismand S. Houinato ${ }^{3}$ \\ ${ }^{1}$ Inter Faculty Center of Training and Research in Environment for Sustainable Development, University of Abomey-Calavi, \\ Cotonou, Benin \\ ${ }^{2}$ Regional Institute of Public Health, University of Abomey-Calavi, Cotonou, Benin \\ ${ }^{3}$ Laboratoire d'Epidémiologie des Maladies Chroniques Et Neurologiques (LEMACEN), Faculty of Health Sciences, Université \\ d'Abomey-Calavi, Cotonou, Benin \\ ${ }^{4}$ Faculty of Humanities and Social Sciences, University of Abomey-Calavi, Cotonou, Benin \\ Email: rochchristianjohnson@gmail.com
}

How to cite this paper: Houinato, A., Johnson, R.C., Kpozehouen, A., Guedou, F., Houssou, C. and Houinato, D.S. (2018) Prevalence of Tobacco Use and Associated Factors among Adults in Benin in 2015: Results of the National Survey of NonCommunicable Disease Risk Factors. Open Journal of Epidemiology, 8, 130-144. https://doi.org/10.4236/ojepi.2018.83011

Received: June 27, 2018

Accepted: August 12, 2018

Published: August 15, 2018

Copyright $\odot 2018$ by authors and Scientific Research Publishing Inc. This work is licensed under the Creative Commons Attribution-NonCommercial International License (CC BY-NC 4.0). http://creativecommons.org/licenses/by-nc/4.0/ c) (i) (8) Open Access

\begin{abstract}
Introduction: Tobacco use and exposure to tobacco smoke are one of the most preventable causes of death and disability worldwide. The risk is even higher among daily tobacco consumers. The World Health Organization (WHO) recommended that surveillance of major risk factors for noncommunicable diseases (NCDs), such as smoking, be imperative to predicting the future burden of NCDs, identifying adequate interventions to decrease future burden and monitor trends. In 2015, a survey on NCD risk factors was conducted in Benin to estimate the prevalence of major NCD risk factors. We analyzed the data from this survey to estimate the prevalence and risk factors associated with daily tobacco consumption in Benin. Methods: A nationwide representative sample of the population was constituted in the 12 departments of Benin. The WHO STEPS wise tool was used to collect data on behavioral and demographic characteristics, including daily tobacco consumption. Tobacco consumption was subdivided into three categories: The daily consumption of tobacco, the daily consumption of smoked tobacco and non-smoked tobacco. Multivariate analysis through the weighted logistic regression was used to identify potential factors associated with daily consumption of tobacco. Results: Over the 5.126 surveyed subjects, $7.49 \%$ were daily tobacco consumers, with $3.85 \%$ being daily users of smoked tobacco and $4.36 \%$ being daily consumers of non-smoked tobacco. Men were more likely
\end{abstract}


to be daily consumers of tobacco compared to women $\left(\mathrm{OR}=7.42 ;{ }_{95 \%} \mathrm{CI}=\right.$ [4.98; 11.06]). Compared to respondents aged 18 to 24 , those aged 45 to 54 were five times more likely to consume tobacco $\left(\mathrm{OR}=5.58 ;{ }_{95 \%} \mathrm{IC}=[2.71\right.$; 11.47]). Respondents residing in the departments of Atacora, Couffo and Borgou were respectively 4.28 times, 3.93 times and 2.63 times more likely to consume tobacco compared to those residing in the department of Alibori. Respondents with no education were more likely to consume tobacco daily compared to respondents who had finished high school or were in university $\left(\mathrm{OR}=3.73 ;{ }_{95 \%} \mathrm{CI}=[1.36 ; 10.23]\right.$. Respondents who were overweight were less likely to be daily users of tobacco compared to people with normal weight status $\left(\mathrm{OR}=0.57 ;{ }_{95 \%} \mathrm{CI}=[0.34 ; 0.96]\right)$. Conclusion: There is a relatively high prevalence of smoking in Benin, where one out of 8 people, consumes tobacco products daily. Being elderly, male, having no formal education, residing in Atacora, Borgou or Couffo were significantly associated with daily smoking. Prevention actions should thus target those sub-groups of the population.

\section{Keywords}

Tobacco, Non Communicable Diseases, STEP Wise, Benin

\section{Introduction}

Tobacco use and exposure to tobacco smoke is one of the most preventable causes of death and disability worldwide. The risk of disability is even higher among daily tobacco users. Compared to occasional smokers, daily smokers are at increased risk of ischemic stroke [1] and serum lipid and lipoprotein concentrations [2]. Projections show that between 2002 and 2030, tobacco-related mortality and exposure are expected to decrease by $9 \%$ in high-income countries, but would double by 3.4 million to 6.8 million in developing countries with low or middle income [3]. The World Report on Non Communicable Diseases (NCDs) 2010 of the World Health Organization (WHO) indicated that in Benin, smoking was a major risk factor for NCDs, which accounted for $16 \%$ of all deaths in the country [4]. WHO recommended that surveillance of the main risk factors for NCDs, such as tobacco use, be imperative in order to predict the future burden of NCDs, identify appropriate interventions to decrease the latter and to monitor current and emerging trends [5]. At present, surveillance of adult smoking in Benin is done through Benin Demographic and Health Surveys (BDHS) [6]. The BDHS provides national data on demographic and health characteristics and contains a section on smoking. However, BDHS only reports prevalence, does not link it to social and demographic characteristics, and does not assess the magnitude of the risk in terms of daily tobacco use. In 2008, the first NCDs risk factors survey was conducted in Benin, involving all 12 departments and using the WHO STEP wise approach, to estimate the prevalence of major risk factors for NCDs. This survey found a relatively high prevalence of 
tobacco consumption: $17.1 \%{ }_{95 \%} \mathrm{CI}=[15.9 ; 18.3]$ for overall tobacco consumption and $9.5 \%{ }_{95 \%} \mathrm{IC}=[8.6 ; 10.5]$ for smoked tobacco [7]. The study also revealed that subjects of male sex, elderly, of low education level, living in rural areas or in some specific departments of the country, were at higher risk of tobacco consumption. This situation has led the national program for NCDs control to strengthen its tobacco consumption prevention activities by targeting these sub-populations. We have used the opportunity of the STEPS survey 2015, as surveillance tool, to assess the current status of these findings by estimating the prevalence of the daily consumption of tobacco and determining potential factors associated to it.

\section{Methods}

A detailed description of the survey design, study population and sampling procedures has been presented elsewhere [8]. However we briefly present here some key features of the methods.

\subsection{Study Design, Study Population and Sampling}

The STEPS survey 2015 was a cross-sectional study conducted between October and December 2015. The target population consisted of adults from the twelve departments of Benin. Inclusion criteria comprised living in Benin for at least 6 months, being aged 18 to 69 years, being present at home on the day of the survey and having provided informed consent. Excluded potential participants included those who were given two unsuccessful home visits as well as those with disabilities (mentally deficient subjects) to answer questions. The sampling framework was provided by the National Institute of Statistics and Economic Analysis (INSAE) based on data from the Fourth General Population and Housing Census (RGPH4) in Benin in 2013. A three-stage random sampling technique was used: the first stage consisted of the random selection of 257 Population Size Estimation Areas (PSEA), the second stage consisted of the randomly selecting 20 households by PSEA and the third stage consisted of randomly selecting one individual per household, using the Kish method recommended by WHO for the STEPS survey. The sample size was calculated using the Scharwtz formula and the spreadsheet developed by the WHO for calculating the sample size in the STEPS surveys. The size of our sample was 5123 participants. The investigation team consisted of 60 trained field researchers and 6 supervisors.

\subsection{The Measurements}

Data were collected following the three steps recommended by WHO in the WHO STEPS methodology and using the latest French version of the WHO STEPS tool adapted for Benin [5]. The first step (STEP 1) consisted of the administration of a questionnaire to obtain information on the demographic, socio-economic and behavioral characteristics of the respondents. At the second 
step (STEP 2), the physical measurements were completed on the respondents. Tobacco consumption (frequency, duration, quantity) was measured under STEP 1. Smoked tobacco products included those when used, can cause the combustion of the tobacco product and its smoke inhalation whereas smokeless tobacco products are those whose consumption involves chewing or snuff.

We considered tobacco users, respondents who reported using smoked tobacco products such as cigarettes, cigars, pipes, shisha and/or smokeless tobacco products such as snuff and tobacco chewing. The daily tobacco users therefore included respondents who declared using on a daily basis, either smoked or smokeless tobacco products or both.

\subsection{Dependent Variable}

The dependent variable was daily tobacco use. In our study, we considered that if a person smoked tobacco or consumed it in any other ways, that person daily consumed tobacco.

\subsection{Independent Variables}

We retained nine (09) variables at the level of the respondents to appreciate factors associated with tobacco consumption. These included 1) gender 2) age, 3) the level of education, 4) marital status, 5) professional status, 6) residence area (rural/urban) 7) department 8) body mass index (BMI) and 8) his waistline. BMI is calculated from the formula IMC $=\frac{\text { Weight }(\mathrm{kg})}{\operatorname{Height}^{2}\left(\mathrm{~m}^{2}\right)}$; WHO thresholds were adopted to define the overall obesity if the BMI $\geq 30$, the overweight if $25 \leq \mathrm{BMI}$ $<30$ and normal weight when the BMI $<25$ [9]. To assess the abdominal obesity we used the waistline of the respondents; the WHO thresholds for the abdominal obesity were used: $94 \mathrm{~cm}$ for men and $80 \mathrm{~cm}$ for women [10] (see Table 1 for the variables and their modalities).

\subsection{Statistical Analysis}

Data were directly captured with Personal Digital Assistant (PDA) devices using the pre-recorded WHO standardized questionnaire for STEPS survey [11].

For the descriptive analysis, proportions were estimated along with their 95\% confidence intervals for the qualitative variables, while central and dispersion parameters were calculated for the quantitative variables.

To identify factors associated with daily smoking, a weighted logistic regression was used to estimate crude and adjusted odds ratios (OR) and their corresponding $95 \%$ confidence intervals $\left({ }_{95 \%} \mathrm{CI}\right)$. Using the significance threshold of $20 \%$, a bivariate analysis was conducted to pre-select variables that were subsequentially included in the multivariate model. These included sex, age, educational attainment, marital status, employment, geographic area of residence, urban/rural residence and weight status. We then used a step down (descending) modeling strategy for the gradual elimination of variables which are less significantly related 
to the daily consumption of tobacco. Variables were retained in the final model if they had a statistical significance level of less than $5 \%$. The weight of the respondents selection was considered for all statistical analyzes [12]. We looked for possible interactions and confounding factors. The fitness of the final model was verified with the test of Hosmer Lemeshow. All statistical analyses were performed using STATA version 12.

\subsection{Ethical Considerations}

The study protocol obtained the approval of the National Ethics Committee for Health Research (CNERS) and the involvement of health authorities and local officials. Free and written informed consent was required from each participant. Data were collected anonymously.

\section{Results}

\subsection{Characteristics of Participants}

A total of 5126 people were surveyed. Women accounted for $50.30 \%$ of this population. Nearly half of the surveyed population (47.30\%) had no level of education. A respondent out of two lived in urban areas (50.70\%) and most of the people surveyed were married or living with a partner $(73.80 \%)$. Two thirds of the respondents (67.70\%) were self-employed and had an independent profession). Their average age was 34.21 years (Standard deviation $=0.31)($ See Table 1$)$.

Table 1. Description of the respondents characteristics in the STEPS Survey, Benin, 2015.

\begin{tabular}{|c|c|c|}
\hline Variable & $\mathrm{n}$ & $\%$ \\
\hline \multicolumn{3}{|l|}{ Sex } \\
\hline Man & 2,326 & 49.70 \\
\hline Woman & 28 & 50.30 \\
\hline \multicolumn{3}{|l|}{ Age (Year) } \\
\hline $18-24$ & 846 & 26.10 \\
\hline $25-34$ & 1,541 & 31.90 \\
\hline $35-44$ & 1,256 & 19.10 \\
\hline $45-54$ & 829 & 13.90 \\
\hline $55-69$ & 654 & 9.05 \\
\hline \multicolumn{3}{|l|}{ Education Level } \\
\hline No formal education & 2,593 & 47.30 \\
\hline Less than primary school & 806 & 15.30 \\
\hline End of primary school & 801 & 15.60 \\
\hline End of high school & 482 & 11.20 \\
\hline End of high school/University & 443 & 10.50 \\
\hline
\end{tabular}




\section{Continued}

\section{Marital status}

Never married

Married/living together

Separated/Divorced

Widower

\section{Professional status}

Employee

Self-employed

Student

Tutor

Volunteer/unemployed/disabled/Retired

3.84

Area

Rural

Urban

Department

$\begin{array}{lcc}\text { Alibori } & 316 & 9.15 \\ \text { Atacora } & 416 & 7.36 \\ \text { Atlantique } & 716 & 13.40 \\ \text { Borgou } & 636 & 12.20 \\ \text { Collines } & 359 & 7.53 \\ \text { Couffo } & 197 & 7.64 \\ \text { Donga } & 299 & 7.46 \\ \text { Littoral } & 339 & 6.96 \\ \text { Mono } & 555 & 5.18 \\ \text { Oueme } & 575 & 9.17 \\ \text { Plateau } & 320 & 6.20 \\ \text { Zou } & 398 & 7.74\end{array}$

\section{Weighted status}

Normal
overweight
Obesity

Waistline

$\begin{array}{lcc}\text { Normal } & 3,926 & 84.40 \\ \text { Abnormal } & 907 & 15.60\end{array}$

n: number of people surveyed. \% weighted columns of respondents. 


\subsection{Prevalence of Tobacco Consumption}

The results of the survey showed that the prevalence of daily smoked tobacco was $3.85 \%{ }_{95 \%} \mathrm{CI}=[3.20 ; 4.50]$, that of daily non smoked tobacco was $4.36 \%$ ${ }_{95 \%} \mathrm{CI}=[3.58 \% ; 5.14 \%]$ and the prevalence of daily consumption of tobacco (smoked or unsmoked) was $7.49 \%{ }_{95 \%} \mathrm{CI}=[6.54 \% ; 8.43 \%]$.

\subsection{Factors Related to Tobacco Use}

\subsubsection{Univariate Analysis: Daily Smoked Tobacco}

Men smoked daily more than women (94.9\% for men versus 5.09\% for women). Men were 20.31 more likely to smoke than women $\left(\mathrm{OR}=20.31 ;{ }_{95 \%} \mathrm{CI}=[10.27\right.$; 40.20]). Respondents smoked mostly in the 55 - 69 age group. A gradual increase in the risk of smoking was noted with increasing age (see Table 2). Respondents with no education were those who smoked the most: the risk of smoking was 3 times higher among respondents without any formal education compared to those who had completed high school or had completed their university education. Separated or divorced respondents were more likely to smoke daily than single $\left(\mathrm{OR}=7.90 ;{ }_{95 \%} \mathrm{CI}=[3.15 ; 19.82]\right)$. The self-employed respondents (independent) were more likely to smoke tobacco daily than the employees (private/public) $\left(\mathrm{OR}=4.83 ;{ }_{95 \%} \mathrm{CI}=[1.53 ; 15.24]\right)$. The departments of Atacora, Couffo and Donga were those where the respondents smoked the most (the respective prevalence were $17.40 \%, 15.80 \%$ and $13.30 \%$ ). Respondents of Atacora were more likely to smoke than those of Alibori $(\mathrm{OR}=2.49$; $\left.{ }_{95 \%} \mathrm{CI}=[1.09 ; 5.69]\right)$, whereas those of Zou or Ouémé were less likely to smoke tobacco (the respective OR were $0.27,{ }_{95 \%} \mathrm{CI}=[0.10 ; 0.76]$ and $0.30 ;{ }_{95 \%} \mathrm{CI}=[0.10$; $0.91])$. Overweight or obese were less likely to smoke than people who had "normal" weight status $\left(\mathrm{OR}=0.42 ;{ }_{95 \%} \mathrm{CI}=[0.24 ; 0.76]\right.$ and $\mathrm{OR}=0.27 ;{ }_{95 \%} \mathrm{CI}=$ $[0.13 ; 0.56]$, respectively; respondents who had a waistline superior to the normal were less likely to daily smoking $\left(\mathrm{OR}=0.13 ;{ }_{95 \%} \mathrm{CI}=[0.05 ; 0.32]\right)$ (see Table 2).

\subsubsection{Univariate Analysis: Daily Non-Smoked Tobacco}

Women consumed more smokeless than smoked tobacco (30\% versus 5.09\%). The older people got, the more they consumed smokeless tobacco; respondents who had no education were more likely to consume non-smoked tobacco compared to those who had completed high school or were in university. Volunteers, unemployed, disabled or retired were more likely to use smokeless tobacco $\left(\mathrm{OR}=10.65 ;{ }_{95 \%} \mathrm{CI}=[1.95 ; 58.12]\right)$ compared to people who had an activity (employees). Respondents from the departments of Couffo, Atacora, Borgou were at higher risk of consuming non-smoked tobacco compared to those from Alibori; the respective ORs were $3.82,2.88,2.18$. Obese people compared to those with normal weight status $\left(\mathrm{OR}=0.34,{ }_{95 \%} \mathrm{CI}=[0.15 ; 0.77]\right)$ and those with an abnormal waistline $\left(\mathrm{OR}=0.49,{ }_{95 \%} \mathrm{CI}=[0.25 ; 0.96]\right)$ were less likely to consume non-smoked tobacco (see Table 2). 
Table 2. Univariate analysis: Demographic and socio-cultural factors related to tobacco.

\begin{tabular}{|c|c|c|c|c|c|c|c|c|c|c|c|c|c|c|c|}
\hline \multirow{3}{*}{ Variable } & \multicolumn{15}{|c|}{ Daily consumption of tobacco } \\
\hline & \multicolumn{5}{|c|}{ Smoked $^{1}$} & \multicolumn{5}{|c|}{ Not - smoked $^{2}$} & \multicolumn{5}{|c|}{ Smoked and/or not - Smoked $^{3}$} \\
\hline & $\mathrm{n}$ & $\%$ & OR & ${ }_{95 \%} \mathrm{CI}$ & p-value & $\mathrm{n}$ & $\%$ & OR & ${ }_{95 \%} \mathrm{CI}$ & p-value & $n$ & $\%$ & OR & ${ }_{95 \%} \mathrm{CI}$ & p-value \\
\hline \multicolumn{16}{|l|}{ Sex } \\
\hline Man & 198 & 94.9 & 20.32 & {$[10.27 ; 40.20]$} & $<0.001$ & 161 & 70.00 & 2.45 & {$[1.59 ; 3.79]$} & $<0.001$ & 335 & 81.40 & 4.91 & {$[3.33 ; 7.24]$} & $<0.001$ \\
\hline Woman & 13 & 5.09 & 1 & - & - & 59 & 30.00 & 1 & - & - & 66 & 18.60 & 1 & - & - \\
\hline \multicolumn{16}{|l|}{ Age } \\
\hline $18-24$ & 8 & 5.31 & 1 & - & - & 11 & 6.57 & 1 & - & - & 19 & 6.56 & 1 & - & - \\
\hline $25-34$ & 54 & 36.5 & 5.83 & {$[2.50 ; 13.59]$} & $<0.001$ & 40 & 26.20 & 3.35 & {$[1.45 ; 7.71]$} & 0.005 & 89 & 31.20 & 4.11 & {$[2.25 ; 7.51]$} & $<0.001$ \\
\hline $35-44$ & 58 & 24.8 & 6.66 & {$[2.88 ; 15.43]$} & $<0.001$ & 57 & 24.80 & 5.40 & {$[2.38 ; 12.26]$} & $<0.001$ & 107 & 25.10 & 5.69 & {$[3.13 ; 10.36]$} & $<0.001$ \\
\hline $45-54$ & 47 & 16.80 & 6.17 & {$[2.61 ; 14.55]$} & $<0.001$ & 69 & 27.60 & 8.49 & {$[3.82 ; 18.86]$} & $<0.001$ & 105 & 22.10 & 7.02 & {$[3.85 ; 12.83]$} & $<0.001$ \\
\hline $55-69$ & 44 & 16.60 & 9.62 & {$[3.99 ; 23.18]$} & $<0.001$ & 43 & 14.80 & 6.90 & {$[2.97 ; 15.99]$} & $<0.001$ & 81 & 15.00 & 7.39 & {$[3.97 ; 13.75]$} & $<0.001$ \\
\hline \multicolumn{16}{|l|}{ Education Level } \\
\hline No formal education & 128 & 60.60 & 3.06 & {$[1.28 ; 7.32]$} & 0.012 & 171 & 74.00 & 47.11 & {$[11.52 ; 192.64]$} & $<0.001$ & 282 & 68.30 & 6.84 & {$[3.00 ; 15.59]$} & $<0.001$ \\
\hline $\begin{array}{c}\text { Less than primary } \\
\text { school }\end{array}$ & 38 & 18.2 & 2.83 & {$[1.10 ; 7.27]$} & 0.031 & 25 & 15.10 & 28.70 & {$[6.50 ; 126.75]$} & $<0.001$ & 56 & 15.40 & 4.59 & {$[1.89 ; 11.12]$} & 0.001 \\
\hline $\begin{array}{c}\text { End of primary } \\
\text { school }\end{array}$ & 24 & 9.92 & 1.48 & {$[0.56 ; 3.89]$} & 0.428 & 15 & 8.75 & 16.06 & {$[3.34 ; 77.20]$} & 0.001 & 37 & 9.53 & 2.69 & {$[1.07 ; 6.81]$} & 0.036 \\
\hline End of high school & 13 & 6.72 & 1.40 & {$[0.48 ; 4.10]$} & 0.543 & 7 & 1.99 & 4.51 & {$[0.78 ; 26.08]$} & 0.092 & 17 & 4.27 & 1.66 & {$[0.60 ; 4.54]$} & 0.327 \\
\hline $\begin{array}{c}\text { End of high } \\
\text { school/University }\end{array}$ & 8 & 4.56 & 1 & - & - & 2 & 0.38 & 1 & - & - & 9 & 2.45 & 1 & - & - \\
\hline \multicolumn{16}{|l|}{ Marital status } \\
\hline Never married & 15 & 7.36 & 1 & - & - & 13 & 9.44 & 1 & - & - & 27 & 8.69 & 1 & & \\
\hline $\begin{array}{l}\text { Married/living } \\
\text { together }\end{array}$ & 173 & 86.10 & 3.51 & {$[1.84 ; 6.71]$} & $<0.001$ & 175 & 80.10 & 2.53 & {$[1.17 ; 5.49]$} & 0.018 & 324 & 82.8 & 2.93 & {$[1.69 ; 5.08]$} & $<0.001$ \\
\hline Separated/Divorced & 14 & 3.69 & 7.90 & {$[3.15 ; 19.82]$} & $<0.001$ & 11 & 4.17 & 7.12 & {$[2.25 ; 22.51]$} & 0.001 & 22 & 3.91 & 7.83 & {$[3.40 ; 18.07]$} & $<0.001$ \\
\hline Widower & 9 & 2.86 & 2.61 & {$[0.92 ; 7.42]$} & 0.71 & 21 & 6.31 & 4.71 & {$[1.84 ; 12.07]$} & 0.001 & 28 & 4.59 & 3.76 & {$[1.81 ; 7.82]$} & $<0.001$ \\
\hline \multicolumn{16}{|l|}{ Professional status } \\
\hline Employee) & 4 & 1.63 & 1 & - & - & 3 & 0.91 & 1 & - & - & 7 & 1.37 & 1 & & \\
\hline Self-employed & 199 & 95.10 & 4.83 & {$[1.53 ; 15.24]$} & 0.007 & 196 & 83.80 & 7.71 & {$[1.90 ; 31.31]$} & 0.004 & 365 & 88.10 & 5.56 & {$[2.27 ; 13.60]$} & $<0.001$ \\
\hline Student & 2 & 1.07 & 0.31 & {$[0.41 ; 2.40]$} & 0.264 & 0 & 0.00 & 1 & - & - & 2 & 0.55 & 0.19 & {$[0.03 ; 1.29]$} & 0.089 \\
\hline Tutor & 2 & 0.56 & 0.15 & {$[0.02 ; 0.94]$} & 0.042 & 13 & 8.86 & 4.53 & {$[0.92 ; 22.29]$} & 0.063 & 15 & 5.45 & 1.83 & {$[0.58 ; 5.81]$} & 0.306 \\
\hline $\begin{array}{l}\text { Volunteer/ } \\
\text { unemployed/ } \\
\text { disabled/ }\end{array}$ & 4 & 1.61 & 1.39 & {$[0.29 ; 6.64]$} & 0.682 & 8 & 6.44 & 10.65 & {$[1.95 ; 58.12]$} & 0.006 & 12 & 4.58 & 5.05 & {$[1.50 ; 16.99]$} & 0.009 \\
\hline \multicolumn{16}{|l|}{ Area } \\
\hline Rural & 132 & 52.90 & 1 & - & - & 135 & 50.70 & 1 & - & - & 253 & 53.20 & 1 & & \\
\hline Urban & 79 & 47.10 & 0.86 & {$[0.60 ; 1.23]$} & 0.411 & 85 & 49.30 & 0.94 & {$[0.65 ; 1.37]$} & 0.764 & 148 & 45.80 & 0.85 & {$[0.64 ; 1.12]$} & 0.240 \\
\hline \multicolumn{16}{|l|}{ Department } \\
\hline Alibori & 10 & 9.18 & 1 & - & - & 12 & 9.36 & 1 & - & - & 19 & 8.35 & 1 & & \\
\hline
\end{tabular}




\section{Continued}

\begin{tabular}{|c|c|c|c|c|c|c|c|c|c|c|c|c|c|c|c|}
\hline Atacora & 45 & 17.40 & 2.49 & {$[1.09 ; 5.69]$} & 0.031 & 62 & 20.00 & 2.88 & {$[1.37 ; 6.02]$} & 0.005 & 99 & 18.80 & 3.23 & {$[1.77 ; 5.90]$} & $<0.001$ \\
\hline Atlantique & 18 & 9.48 & 0.70 & {$[0.28 ; 1.72]$} & 0.432 & 3 & 1.30 & 0.09 & {$[0.02 ; 0.39]$} & 0.001 & 21 & 5.63 & 0.44 & {$[0.21 ; 0.91]$} & 0.027 \\
\hline Borgou & 29 & 11.00 & 0.90 & {$[0.38 ; 2.12]$} & 0.802 & 67 & 25.70 & 2.18 & {$[1.03 ; 4.60]$} & 0.042 & 94 & 20.40 & 1.96 & {$[1.06 ; 3.61]$} & 0.032 \\
\hline Collines & 13 & 4.46 & 0.58 & {$[0.21 ; 1.59]$} & 0.291 & 12 & 4.01 & 0.51 & {$[0.21 ; 1.26]$} & 0.146 & 24 & 4.56 & 0.65 & {$[0.32 ; 1.33]$} & 0.234 \\
\hline Couffo & 15 & 15.80 & 2.16 & {$[0.85 ; 5.51]$} & 0.108 & 26 & 26.50 & 3.82 & {$[1.69 ; 8.62]$} & 0.001 & 35 & 20.20 & 3.36 & {$[1.70 ; 6.64]$} & $<0.001$ \\
\hline Donga & 19 & 13.30 & 1.83 & {$[0.73 ; 4.63]$} & 0.200 & 17 & 8.18 & 1.07 & {$[0.44 ; 2.61]$} & 0.875 & 34 & 10.50 & 1.60 & {$[0.80 ; 3.21]$} & 0.180 \\
\hline Littoral & 8 & 3.05 & 0.43 & {$[0.14 ; 1.26]$} & 0.124 & 1 & 0.33 & 0.04 & {$[0.01 ; 0.35]$} & 0.003 & 8 & 1.57 & 0.23 & {$[0.09 ; 0.61]$} & 0.003 \\
\hline Mono & 24 & 4.91 & 0.94 & {$[0.39 ; 2.26]$} & 0.894 & 15 & 3.13 & 0.58 & {$[0.24 ; 1.39]$} & 0.220 & 33 & 3.35 & 0.69 & {$[0.36 ; 1.35]$} & 0.282 \\
\hline Oueme & 7 & 2.84 & 0.30 & {$[0.10 ; 0.91]$} & 0.034 & 1 & 0.56 & 0.06 & {$[0.01 ; 0.45]$} & 0.007 & 8 & 1.79 & 0.20 & {$[0.08 ; 0.51]$} & 0.001 \\
\hline Plateau & 14 & 6.39 & 1.03 & {$[0.38 ; 2.76]$} & 0.955 & 2 & 0.55 & 0.08 & {$[0.02 ; 0.39]$} & 0.002 & 16 & 3.60 & 0.62 & {$[0.28 ; 1.39]$} & 0.247 \\
\hline Zou & 9 & 2.19 & 0.27 & {$[0.10 ; 0.76]$} & 0.013 & 2 & 0.38 & 0.05 & {$[0.01 ; 0.21]$} & $<0.001$ & 10 & 1.23 & 0.16 & {$[0.07 ; 0.38]$} & $<0.001$ \\
\hline \multicolumn{16}{|c|}{ Weight status } \\
\hline Normal & 181 & 86.00 & 1 & - & - & 187 & 81.70 & 1 & - & - & 341 & 82.90 & 1 & - & - \\
\hline overweight & 18 & 9.07 & 0.42 & {$[0.24 ; 0.76]$} & 0.004 & 21 & 12.50 & 0.62 & {$[0.33 ; 1.19]$} & 0.150 & 37 & 11.80 & 0.56 & {$[0.35 ; 0.90]$} & 0.016 \\
\hline Obesity & 12 & 4.88 & 0.27 & {$[0.13 ; 0.56]$} & $<0.001$ & 12 & 5.80 & 0.34 & {$[0.15 ; 0.77]$} & 0.037 & 23 & 5.30 & 0.30 & {$[0.17 ; 0.53]$} & $<0.001$ \\
\hline \multicolumn{16}{|l|}{ Waistline } \\
\hline Normal & 200 & 97.60 & 1 & - & - & 197 & 91.50 & 1 & - & - & 370 & 94.40 & 1 & - & - \\
\hline Abnormal & 7 & 2.36 & 0.13 & {$[0.05 ; 0.32]$} & $<0.001$ & 18 & 8.50 & 0.49 & {$[0.25 ; 0.96]$} & 0.037 & 23 & 5.58 & 0.30 & {$[0.16 ; 0.54]$} & $<0.001$ \\
\hline
\end{tabular}

${ }^{1}$ Smoked: Product of tobacco that gives smoke during consumption (cigarette, cigars, cigarillos, hand rolled cigarettes). ${ }^{2}$ Not-smoked: Tobacco product that is consumed in any other forms without smoke (chewing, snuff). ${ }^{3}$ Smoked and/or Not Smoked: Tobacco in general consumed in all its forms. n: number of people surveyed. \%: proportion of respondents who consume tobacco. OR: Odds Ratio.

\subsubsection{Univariate Analysis: Daily Smoked or Non-Smoked Tobacco (SONST)}

Trends observed among respondents for the consumption of smoked or non-smoked tobacco were noted for SONST consumption. The departments in which people consumed more SONST were Borgou (20.40\%), Couffo (20.20\%), Atacora (18.80\%), and Donga (10.50\%). The risk of daily consumption of tobacco in all its forms was lower in the Atlantique Departments $(\mathrm{OR}=0.44$; $\left.{ }_{95 \%} \mathrm{CI}=[0.21 ; 0.91]\right)$, Littoral $\left(\mathrm{OR}=0.23 ;{ }_{95 \%} \mathrm{CI}=[0.09 ; 0.61]\right)$, Ouémé $(\mathrm{OR}=$ $\left.0.20 ;{ }_{95 \%} \mathrm{CI}=[0.08 ; 0.51]\right)$ and that of $\mathrm{Zou}\left(\mathrm{OR}=0.16 ;{ }_{95 \%} \mathrm{CI}=[0.07 ; 0.38]\right)$ in relation to the department of Alibori. Overweight or obese people were less likely to consume SONST; the respective ORs were $0.56 ;{ }_{95 \%} \mathrm{CI}=[0.35 ; 0.90]$ and 0.30 ; ${ }_{95 \%} \mathrm{CI}=[0.17 ; 0.53]$. Respondents who had an abnormal waistline were less likely to consume SONST $\left(\mathrm{OR}=0.30 ;{ }_{95 \%} \mathrm{CI}=[0.16 ; 0.534]\right)$ (see Table 2$)$.

\subsubsection{Multivariate Analysis: Daily Smoked or Non-Smoked Tobacco (SONST)}

In multivariate analysis, men were more at risk of consuming SONST than women $\left(\mathrm{OR}=7.42{ }_{95 \%} \mathrm{CI}=[4.98 ; 11.06]\right.$. Compared to respondents who were in the 18 to 24 age group, respondents who belonged to other age groups were more likely to consume SONST (see Table 3). Respondents with no education 
Table 3. Multivariate analysis: Demographic and socio-cultural factors related to tobacco consumption in Benin, STEP survey, 2015.

\begin{tabular}{|c|c|c|c|}
\hline \multirow{2}{*}{ Variables } & \multicolumn{3}{|c|}{ Tobacco consumption (in all its forms) } \\
\hline & OR & ${ }_{95 \%} \mathrm{CI}$ & P-value \\
\hline \multicolumn{4}{|l|}{ Sex } \\
\hline Man & 7.42 & {$[4.98 ; 11.06]$} & $<0.001$ \\
\hline Woman & 1 & & \\
\hline \multicolumn{4}{|l|}{ Age } \\
\hline $18-24$ & 1 & & \\
\hline $25-34$ & 3.55 & {$[1.84 ; 6.84]$} & $<0.001$ \\
\hline $35-44$ & 4.31 & {$[2.14 ; 8.70]$} & $<0.001$ \\
\hline $45-54$ & 5.58 & {$[2.71 ; 11.47]$} & $<0.001$ \\
\hline $55-69$ & 4.48 & {$[2.19 ; 9.17]$} & $<0.001$ \\
\hline \multicolumn{4}{|l|}{ Education Level } \\
\hline No formaleducation & 3.73 & {$[1.36 ; 10.23]$} & 0.011 \\
\hline Less than primary school & 2.89 & {$[0.99 ; 8.43]$} & 0.051 \\
\hline End of primary school & 1.47 & {$[0.47 ; 4.56]$} & 0.506 \\
\hline End of high school & 1.22 & {$[0.40 ; 3.72]$} & 0.721 \\
\hline End of high school/University & 1 & & \\
\hline \multicolumn{4}{|l|}{ Marital status } \\
\hline Never married & 1 & & \\
\hline Married/living together & 1.42 & {$[0.77 ; 2.62]$} & 0.261 \\
\hline Separated/Divorced & 2.81 & {$[1.01 ; 7.83]$} & 0.049 \\
\hline Widower & 2.33 & {$[0.89 ; 6.11]$} & 0.085 \\
\hline \multicolumn{4}{|l|}{ Professional status } \\
\hline Employee & 1 & & \\
\hline Self-employed & 3.17 & {$[1.00 ; 10.13]$} & 0.051 \\
\hline Student & 0.58 & {$[0.08 ; 4.52]$} & 0.606 \\
\hline Tutor & 3.03 & {$[0.74 ; 12.49]$} & 0.125 \\
\hline Volunteer/unemployed/disabled/Retired & 6.60 & {$[1.63 ; 26.75]$} & 0.008 \\
\hline \multicolumn{4}{|l|}{ Area } \\
\hline Rural & 1 & & \\
\hline Urban & 1.32 & {$[0.95 ; 1.82]$} & 0.093 \\
\hline \multicolumn{4}{|l|}{ Department } \\
\hline Alibori & 1 & & \\
\hline Atacora & 4.28 & {$[2.24 ; 8.18]$} & $<0.001$ \\
\hline Atlantic & 0.62 & {$[0.30 ; 1.30]$} & 0.207 \\
\hline Borgou & 2.63 & {$[1.39 ; 4.98]$} & 0.003 \\
\hline Collines & 0.97 & {$[0.44 ; 2.11]$} & 0.932 \\
\hline
\end{tabular}




\begin{tabular}{lccc} 
Continued & & & \\
\hline Couffo & 3.93 & {$[1.88 ; 8.24]$} & $<0.01$ \\
Donga & 1.79 & {$[0.87 ; 3.69]$} & 0.115 \\
Littoral & 0.33 & {$[0.12 ; 0.92]$} & 0.034 \\
Mono & 0.82 & {$[0.40 ; 1.66]$} & 0.577 \\
Oueme & 0.22 & {$[0.09 ; 0.58]$} & 0.002 \\
Plateau & 0.58 & {$[0.25 ; 1.35]$} & 0.204 \\
Zou & 0.17 & {$[0.07 ; 0.41]$} & $<0.001$ \\
Weighted status & & & \\
Normal & 1 & & 0.034 \\
overweight & 0.57 & {$[0.34 ; 0.96]$} & 0.281 \\
Obesity & 0.71 & {$[0.38 ; 1.32]$} & \\
\hline
\end{tabular}

were more likely to consume SONST than those who had been to high school or university $\left(\mathrm{OR}=3.73 ;{ }_{95 \%} \mathrm{CI}=[1.36 ; 10.23]\right.$. Divorced people were more likely to use the SONST compared to respondents who have never been married. Volunteers, unemployed, disabled or retired were 7 times more likely to consume SONST than those who worked (public or private employees). Compared to the department of Alibori, respondents in the departments of Atacora, Borgou, Couffo were more at risk of consuming SONST. The overweight people were less at risk of consuming SONST $\left(\mathrm{OR}=0.71{ }_{95 \%} \mathrm{CI}=[0.38 ; 1.32]\right.$ than those who had a normal BMI (See Table 3).

\section{Discussion}

\subsection{Achievement of the Purpose of the Study}

The prevalence of daily smoked tobacco was $3.85 \%{ }_{95 \%} \mathrm{CI}=[3.20 ; 4.50]$, that of daily non-smoked tobacco was $4.36 \%{ }_{95 \%} \mathrm{CI}=[3.58$; 5.14$]$ and that of daily tobacco consumption (smoked or non-smoked) was $7.49 \%{ }_{95 \%} \mathrm{CI}=[6.54 ; 8.43]$.

From our analyses, factors related to tobacco consumption included being: male subjects ( $\mathrm{p}<0.001)$; aged from 18 to $69(\mathrm{p}<0.001)$; with no education $(\mathrm{p}=$ $0.011)$; separate or divorced ( $\mathrm{p}=0.049)$; volunteers, unemployed, disabled or retired $(\mathrm{p}=0.008$ and from the departments of Atacora $(\mathrm{p}<0.001)$.

Variables such as living environment (urban or rural), weight status, and waistline were not significantly associated with daily smoking.

\subsection{Validity of Results}

The methodology used is consistent with that recommended by WHO for this type of study. The size of our sample was large enough for the results to be generalizable for the Beninese population. Also, the use of a standardized questionnaire means that the results obtained could be compared to those of other countries. A limitation of this study was its cross-sectional design that could not allow any temporal relationship. Another limitation was the definition of the va- 
riable "daily consumption of tobacco," which excluded occasional tobacco users and could therefore underestimate the extent of smoking in Benin. Alternative variable for WHO STEPS survey was "current smoking" which did not either specify the duration, was vague and subject to interpretations from the respondents. Future STEPS surveys should include a time component in the assessment of "current smoking". In addition, the evaluation of the "daily tobacco consumption" was based on self-reports that could introduce a bias. Finally, the present study was not able to cover the passive tobacco consumption, which in fact, is quite difficult to measure.

\subsection{Comparison of Results}

\subsubsection{Overall Prevalence}

The overall prevalence of daily tobacco consumption in Benin during our study was higher than that obtained in the Democratic Republic of Congo (DRC) in 2005 when a STEPS survey conducted among subjects aged from 15 to 64 found 4.4\% [13]. It was also higher than that found in the STEPS survey in Ethiopia in 2006, (4.6\%) among individuals aged from 25 to 64 [14] and that found in the STEPS survey 2008 in Zambia in adults over the age of 25 (5.0\%) [15]. These differences could be explained by the difference between the sizes of the age groups considered. The prevalence of daily tobacco consumption was lower than that found in Benin in 2008 [16] during the STEPS survey., This reduction in prevalence could be explained by the various interventions conducted by the Ministry of Health and civil society for the dissemination of the STEPS survey 2008 results. The prevalence was also lower than that found in 2014 in Uganda during a national survey (9.2\%) [17], in Tanzania (15.9\%) in 2012 [18] and in Mozambique (16.7\%) in 2005 [19] during the STEPS investigation on subjects aged from 25 to 64. In 2017, in France, the prevalence of daily smoking was $26.9 \%$ [20]. A study conducted among students in Syria in 2010 also showed a higher prevalence of $20.75 \%$ [21]. The difference could be explained by the difference in study populations.

\subsubsection{Prevalence by Sex}

The male predominance observed in our study was also found in the STEPS survey in Benin in 2008 (22.5\% vs. 5.9\%) [16], in 2014 in Uganda during a national demographic survey (15.9\% vs. 4.7\%) [17] and in the DRC in 2005 during the STEPS survey (14.1\% vs. 1.4\%) [13]. This predominance could be explained by the fact that in African culture, the education given to girls does not allow them to smoke, not even to declare it. They are therefore less likely to smoke than boys and even the majority of those who smoke will not always be able to declare it during the surveys because they are afraid of the judgment of others. In contrast, the study conducted in the United States and South Korea found no link between gender and smoking [22]. This difference could be explained by the difference between the target populations. In fact the study only targeted teenagers while ours has considered all the general population. Also, the difference 
between the results can also be explained by the fact that in the culture of the Western people, there is no blame in society about women who smoke. They are therefore free as much as men to smoke.

\subsubsection{Prevalence by Age}

The age group of adults who were the most at risk in our study was similar to that found in Nepal in 2011, where the age group from 36 to 49 was concerned by smoking [23]. It was also similar to that found in Uganda in 2014 (30 to 49 years old) [17]. National studies carried out in Benin in 2008 [16], in Togo in 2010 [24] and in Burkina Faso in 2013 [25], found an age group at risk higher than that of our study (55 to 64 years old). This trend of predominance of tobacco use among older adults could be explained by the fact that most anti-smoking activities are directed towards young people, hence the reduction of prevalence among the latter. It should be added that during the rites in Africa, tobacco is used and mostly by elderly persons, which could explain our results.

\subsubsection{Prevalence by Level of Education}

The link between low education and smoking was also found in a study conducted in Pakistan in 2004 and 2005 [26]. The National Population Survey conducted in Nepal in 2011 also found the same result $(p<0.01)$ [23]. This could be explained by the fact that educated individuals would be more aware of the consequences of smoking and be wary of non-educated individuals.

\subsubsection{Prevalence by Occupation}

The fact that people who do not have full-time jobs are more at risk of smoking is similar to the observation made in Togo in 2010 during the STEPS survey [24]. This could be explained by the fact that unemployment leads individuals to smoking to forget the problems linked to their status. The type of occupation was linked to smoking in France in 2014. Laborers smoked more daily than other workers and employees smoked less [27]. This same remark was made in Nepal in 2011, during the demographic survey. This difference could be explained by the difference in methods of study. It could also be explained by the fact that occupations that require a lot of physical effort are quite stressful, so the workers indulge in smoking to better manage stress.

\subsubsection{Prevalence According to Marital Status}

In contrast to our study, the demographic survey conducted in Nepal in 2011 showed that married couples were more at risk of using tobacco [27]. $(\mathrm{p}<0.01)$. The difference could also be explained by the difference between the methods used. However, our results could be supported by the fact that, being divorced, people tend to smoke to cope with problems related to such a status.

\section{Conclusions}

There is a high prevalence of daily smoking in Benin with nearly one out of 8 people using tobacco daily. Being old, being of a male gender, having no formal 
education, residing in Atacora, Borgou or Couffo were significantly and positively linked to daily smoking while being overweight were inversely associated with it.

The results of this study provide useful information to the National Program against smoking for public health interventions designed for the control and the prevention of smoking in Benin.

\section{Competing Interests}

The authors state that they have no competing interests.

\section{References}

[1] Bhat, V.M., Cole, J.W., Sorkin, J.D., Wozniak, M.A., Malarcher, A.M., Giles, W.H., et al. (2008) Dose-Response Relationship between Smoking and Risk of Ischemic Stroke in Young Women. Stroke, 39, 2439-2443.

https://doi.org/10.1161/STROKEAHA.107.510073

[2] Craig, W.Y., Palomaki, G.E. and Haddow, J.E. (1989) Cigarette Smoking and Serum Lipid and Lipoprotein Concentrations: An Analysis of Published Data. BMJ, 298, 784-788. https://doi.org/10.1136/bmj.298.6676.784

[3] Castellsague, X., Munoz, N., Destefani, E., Victora, C.G., Castelletto, R., Rolon, P.A., et al. (1999) Independent and Joint Effects of Tobacco Smoking and Alcohol Drinking on the Risk of Esophageal Cancer in Men and Women. International Journal of Cancer, 82, 657-664. https://doi.org/10.1002/(SICI)1097-0215(19990827)82:5<657::AID-IJC7>3.0.CO;2-C

[4] WHO (2010) Global Status Report on Noncommunicable Diseases. http://www.who.int/nmh/publications/ncd_report_full_en.pdf

[5] World Health Organization (2015) STEPwise Approach to Monitoring (STEPS). http://www.who.int/chp/steps/en/

[6] INSAE and ICF International (2013) National Institute of Statistics and Economic Analysis (INSAE) and ICF International. Demographic and Health Survey (EDSB IV) of Benin 2011-2012. Calverton, MD.

[7] Nubukpo, P., Gbary, A.R., Ouendo, E.M., Kounouhehoua, N., Segnon, J., Laqueille, X., et al. (2012) Smoking in the General Population in Benin. Alcoology and Addictology, 34, 265-272.

[8] Ministry of Health (2016) Final Report of the Survey for the Surveillance of Risk Factors for Noncommunicable Diseases using the STEPSwise Approach of the WHO STEPS 2015 SURVEY in Benin. National Program for the Control of Noncommunicable Diseases.

[9] World Health Organization. Consultation on obesity Classification according to Body Mass Index.Geneva; 1993.

[10] World Health Organization (2008) Waist Circumference and Waist-Hip Ratio: Report of a WHO Expert Consultation. WHO, Geneva. http://apps.who.int/iris/bitstream/handle/10665/44583/9789241501491_eng.pdf;jses sionid $=84221925$ D95DDECB187F2454821

[11] World Health Organization (2014) WHO STEPS Questionnaire (Basic and Expanded Module).

[12] Bennett, S., Woods, T., Liyanage, W. and Smith, D. (1991) A Simplified General Method for Cluster-Sample Surveys of Health in Developing Countries. World 
Health Statistics Quarterly, 44, 98-106.

[13] Longo, M. (2005) Democratic Republic of Congo (City of Kinshasa) STEPS Survey 2005 Factsheet. http://www.who.int/chp/steps/2005_DRC_FactSheet_EN.pdf

[14] Tesfaye, F. (2003) Ethiopia (Butajira) STEPS Survey 2003 Fact Sheet. http://www.who.int/chp/steps/2006_Ethiopia_FactSheet_EN.pdf

[15] Zambia Ministry of Health, World Health Organization Country Office (2008) Prevalence Rates of the Common Noncommunicable Disease Risk Factors in Lusaka District, Zambia. http://www.who.int/chp/steps/2008_Zambia_FactSheet_EN.pdf?Ua=1

[16] Ministry of Health (2008) Final Report of the Survey for the Surveillance of Risk Factors for Non Communicable Diseases Using the STEPSwise Approach of WHO Survey "STEPS 2008" in Benin. National Program for the Control of Non Communicable Diseases.

[17] Kabwama, S.N., Ndyanabangi, S., Mutungi, G., Wesonga, R., Bahendeka, S.K. and Guwatudde, D. (2016) Tobacco Use and Associated Factors among Adults in Uganda: Findings from a Nationwide Survey. Tobacco Induced Diseases, 261, 1169-1173. https://doi.org/10.1186/s12971-016-0093-8

[18] Mayige, M. (2012) Tanzania STEPS Survey 2012 Fact. http://www.who.int/chp/steps/UR_Tanzania_FactSheet_2012.pdf?ua $=1$

[19] Damasceno, A.A. (2005) Mozambique STEPS Survey 2005 Factsheet. http://www.who.int/chp/steps/2005_Mozambique_FactSheet_EN.pdf?u=1

[20] Public Health France (2016) Weekly Epidemiological Bulletin.

[21] Al-Kubaisya, W., Abdullaha, N., Al-Nuaimya, H., MuzaKahnb, S., Halawanyc, G. and Kurdyc, S. (2010) Factors Associated with Smoking Behavior among University Students in Syria Pacific. International Conference on Environment-Behavior Studies, Kuching, December 2010.

[22] Sung, S.C. and Kyoung, H. (2014) Risk Factors for Current Smoking among American and South Korean Adolescents, 2005-2011. Clinical Scholarship, 46, 408-415. https://doi.org/10.1111/jnu.12099

[23] Khanal, V., Adhikari, M. and Karki, S. (2013) Social Determinants of Tobacco Consumption among Nepalese Men: Findings from Nepal Demographic and Health Survey 20011. Harm Education Journal, 10, 10.

[24] Ministry of Health (2010) Final Report of the STEPS TOGO 2010 Survey. Non-Communicable Diseases Department of Division and Epidemiology.

[25] Ministry of Health (2013) Report of the National Survey on the Prevalence of the Main Common Risk Factors for Non Communicable Diseases in Burkina Faso STEPS 2013 Survey.

[26] Ali, Y.A., Azhar, I., Khalif, B.M., Ronald, E.M., Ashfaq, A. and Sania, M. (2008) Investigating Socio-Economic-Demographic Determinants of Tobacco Use in Rawalpindi, Pakistan. BMC Public Health, 8, 50.

[27] Guignard, R., Beck, F., Richard, J.-B., Lermenier, A., Wilkin, J.L. and NguyenThanh, V. (2015) Smoking in France in 2014: Characteristics and Recent Evolutions. Evolutions Results of Studies and Research in Prevention and Health Education, 31, 1-6. 